\title{
Introduction to Special Issue
}

\section{Community Integration Following SCI}

\author{
William O. McKinley and Michelle A. Meade ${ }^{\mathrm{b}}$

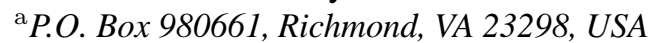 \\ Tel.: +1 804828 4233; Fax: +1 804828 5074; E-mail: wmckinle@hsc.vcu.edu. \\ ${ }^{\mathrm{b}}$ Virginia Commonwealth University, MCV Campus, PO Box 980677, Richmond, VA 23298-0677, USA \\ Tel.: +1 804828 5401; Fax: +1 804828 6340; E-mail: mameade@vcu.edu
}

Spinal Cord Injury (SCI) can result in long-term physical impairments, subsequent disability (inability to perform functional activities) and difficulties participating in social activities. For individuals with this condition, successful rehabilitation involves maximizing community integration areas, including: the ability to participate in community life activities, remain active in family and recreational events, and access local activities and resources, including health care followup and employment opportunities. Community integration emphasizes the ability of individuals to overcome disability barriers and has been shown to have a positive influence on their quality of life. With recent health care trends emphasizing earlier discharges from acute rehabilitation centers, it becomes increasingly important to adequately prepare individuals (and their support networks) for return to home and community.

This special issue of NeuroRehabilitation presents nine articles that address this continuum of community integration. Prominent researchers associated with the National Institute on Disability and Rehabilitation Research (NIDRR) and the National Spinal Cord Injury Model Systems have submitted articles that provide state-of-the art research and review of the current literature related to this topic. Three key "layers" of the community integration continuum will be highlighted in this issue: the relationship of participation to personal characteristics, the effects of the environment and the benefits of activity-based community opportunities.

At the first layer, the impact of personal characteristics is considered. Personal characteristics range from race and gender to personality variables and motivation. In this issue, Krause and his colleagues examine how gender and race impact on psychosocial and quality of life outcomes. Their outcomes highlight the importance of personal characteristics in determining reported priorities and areas of need associated with life satisfaction and participation. In a related article, Charlifue and Gerhart examine how aging is associated with community integration for persons with SCI. The findings indicate a general decline in community reintegration over time in terms of physical independence, mobility, occupation and social integration with an associated decline in life satisfaction.

The next layer affecting participation is environmental characteristics that can serve as barriers to, or supports for, activities and independence. This layer includes factors such as the physical and social environment, community resources, and public policy. Centers for Independent Living (CILs) are community-based programs that help to provide necessary resources and minimize barriers to enable this process to be more effective. The article by Forchheimer and Tate discusses the need for a variety of independent living skills and the association of those skills with outcomes while highlighting the importance of communication between medical centers and CILs. Personal assistant services, another support that allow persons with SCI to achieve greater independence and community integration, are reviewed by Hagglund and his colleagues. After discussing the benefits and importance of these services, evidence-based recommendations are made with regard 
to both policy and future research amongst persons with SCI.

Finally, there are the activities themselves, and the benefits that they offer to those who engage in them. In this special issue, Slater and Meade discuss the benefits of recreation and sports for persons with SCI. This type of community integration highlights the physical and psychosocial benefits associated with participation without the complicated interactions of policy and work supports and disincentives. Though recreation or play is sometimes viewed as a pre-vocational activity, its importance for promoting both physical and emotional health is emphasized.

Often viewed as the pinnacle of community integration, employment has been associated with a sense of productivity, self-sufficiency and increased life satisfaction. Too often rates for employment for persons with SCI are alarmingly low, even for those employed at the time of injury. The topic of employment for persons with SCI is explored in four articles that discuss supports, assistive technology and opportunities. Targett and colleagues discuss the myriad of challenges faced by persons with SCI such as lack of transportation, limited accessibility, and health problems. They describe how a Supported Employment approach can be used to assist persons with SCI with return to work. McKinley and colleagues continue the exploration of vocational supports with a review of the literature on the ability of assistive technology to compensate for functional limitations, enhance technical capacities and overcome barriers to employability following SCI. These accommo- dations are particularly relevant for persons with SCI given the likelihood that employment opportunities will include more sedentary type positions (as opposed to manual labor positions) and the need for enhanced education. Bricout discusses telework as an option for persons with Spinal Cord Injury. By using Information and Communication Technologies (ICT) to allow individuals to work from their homes, this option has the potential to alleviate many of the barriers associated with working that persons with SCI face. Finally, the potential of a prototype advanced mobility device (IBOT 3000) to overcome transportation and mobility barriers is discussed in the final article of this issue. Cooper and his colleagues report on the mobility and satisfaction of individuals utilizing the IBOT 3000 to access to community activities, social events and personal/professional appointments within their community. They note that enhanced accessibility may be associated with increased satisfaction with performance.

Taken together, these articles reflect not only our increasing understanding of the issues impacting participation following SCI, but also the varied approaches that have been utilized to facilitate the process. While the creativity and dedication of both researchers and activists have increased the abilities of and opportunities for persons with SCI to engage in activities, vigilance must be maintained to make these the rule rather than the exception. Community integration, and the necessary supports and accommodations for it, still need to be incorporated into public consciousness as rights rather than research projects or luxuries. 